\title{
Design and Parametric Variation Assessment of Dopingless Nanotube Field-Effect Transistor (DL-NT- FET) for High Performance
}

Jeetendra Singh ( $\sim$ jeetendras15.phd@nitj.ac.in )

NIT Sikkim: National Institute of Technology Sikkim

Debapriya Chakraborty

NIT Sikkim: National Institute of Technology Sikkim

Naveen Kumar

NIT Jalandhar: Dr BR Ambedkar National Institute of Technology

\section{Research Article}

Keywords: nanotube FET, Dopingless, charge plasma, output conductance, average subthreshold slope, and gate capacitance

Posted Date: April 12th, 2021

DOI: https://doi.org/10.21203/rs.3.rs-330678/v1

License: (9) This work is licensed under a Creative Commons Attribution 4.0 International License. Read Full License 


\section{Abstract}

In this paper, a dopingless nanotube field-effect transistor (DL-NT-FET) has been proposed and its performance analysis is done using a technology computer-aided design (TCAD) tool, ATLAS provided by Silvaco. The elimination of doping is brought in by the application of the charge-plasma (CP) technique. A comparative examination of transfer characteristics (I D -V GS ), transconductance ( $\mathrm{g} \mathrm{m}$ ), gate capacitances ( $\mathrm{C}$ gs and C gd), output characteristics (I D -V DS ), output conductance ( $\mathrm{g}$ ds ), average subthreshold slope (AVSS), the threshold voltage ( $V t$ ), the ratio of on-current to off-current (I ON /I OFF ) and on-current has been made by varying the channel length $(\mathrm{Lg})$, radius $(R)$, gate work function $(\Phi)$, and temperature. Results revealed that increasing the channel length improves subthreshold slope with greater I ON /I OFF and less threshold voltage. It has been also noticed that increase in the radius of the nanotube or an increase in temperature results in just the opposite effect of that observed in the case of increasing channel length. The I OFF value increases significantly on increasing the temperature while the small degradation in the I ON has been noticed as a result of mobility degradation and velocity saturation. The I ON degrades $15 \%$ by increasing the temperature from $200 \mathrm{~K}$ to $400 \mathrm{~K}$. The output conductance $\mathrm{g}$ ds also degrades on increasing the temperature. A proliferation of $39 \%$ is observed in the $\mathrm{C}$ gs at the V GS of $0.45 \mathrm{~V}$ on increasing the channel length from $20 \mathrm{~nm}$ to $35 \mathrm{~m}$ whereas no significant changes are observed in the $\mathrm{C}$ gd for the same increment in the channel length.

\section{Introduction}

The complementary metal-oxide-semiconductor (CMOS) technology has advanced through the decades to dominate the semiconductor industry with excellent features of low power and cost, dense packaging, and high-speed devices that are continuously scaled down in size [1-3]. When it comes to designing $\mathrm{RF} /$ analog circuits in the nanometer regime, several challenges have been encountered due to strict process requirements to maintain sharp source/drain region and short channel effects (SCEs) [4-7]. The junctionless field-effect transistor (JLFET) solves many of the issues related to SCEs but still, it suffers from poor carrier mobility due to high channel doping, which leads to transconductance/gain degradation and hence low ON current $[8,9]$. Dopingless nanotube FET (DL-NT-FET) can provide a solution by making use of the charge-plasma (CP) technique in which abrupt doped source and drain regions are formed in the intrinsic substrate with the help of suitable work function metal electrode or poly-Si electrode at the high temperature [10-12]. The gate-all-around (GAA) and shell-gated nanotube provide strong gate control over the channel carrier which results in enhanced surface inversion and hence superior drain current [13, 14]. Hence, the proposed device exploits both the advantages of GAA structure (i.e. allowing better electrostatics and reduced SCEs) and dopingless configuration (like reduced random dopant fluctuations(RDFs), less mobility degradation, better gm) $[15,16]$. Besides the collective merit of GAA and dopingless nanotube, the impact of temperature on the performance parameters of the proposed DL-NTFET is the first time investigated along with other design parameters.

In this article, a dopingless nanotube field-effect transistor (DL-NT-FET) is designed and its various performance parameters; transfer characteristics (ID-VGS), transconductance (gm), gate capacitances 
(Cgs and Cgd), output characteristics (ID-VDS), output conductance (gds), average subthreshold slope (AVSS), the threshold voltage (Vt), the ratio of on-current to off-current (ION/IOFF) are investigated. The impact of nanotube radius $(\mathrm{R})$, channel length $(\mathrm{Lg})$, and gate work function variations on the performance parameters are examined to achieve high performance. The various performance parameters are also evaluated at a different temperature to study the temperature-dependent variability.

\section{Device Structure}

The 2-D structure of the proposed device is shown in Fig. 1(a) whereas the 3-D structure of the same is depicted in Fig. 1(b). The source, channel, and drain regions have been formed on an intrinsic Si body with the radius being $10 \mathrm{~nm}$ and the silicon thickness being $7 \mathrm{~nm}$ leaving the inner oxide thickness. CP technique is used to establish the source and drain regions of this dopingless device [17]. The gate oxide is made of $\mathrm{SiO} 2$ and is $2 \mathrm{~nm}$ in thickness. Both drain and source lengths are $30 \mathrm{~nm}$ while the channel length is $20 \mathrm{~nm}$. P-type polysilicon (workfunction $=3.9 \mathrm{eV}$ ) is used in forming the source and drain coreelectrodes. The gate contact is also made of polysilicon (workfunction=4.6eV). The poly-Silicon coreelectrodes help to induce $\mathrm{N}+$ regions at the source and drain sides. A SiO2 layer surrounds these electrodes followed by an intrinsic Silicon layer in the core. Poly-silicon electrodes are also attached at the bottom and top of this layer. The second layer of SiO2 is muffled on all sides of the Si layer. A shell consisting of polysilicon (work function $=4.6 \mathrm{eV}$ ) has been wrapped over the oxide layer to form a contact for the gate. Aluminum is generally preferred for gate material of MOSFET but here the polysilicon is mainly utilized because polysilicon composition matches with the channel bulk-silicon and therefore offers low threshold voltage as compared to that of metal gate material [18]. The proposed device is simulated utilizing Silvaco TCAD to analyze the various results. To account for accurate mobility evaluation in the channel, the Lombardi mobility model along with concentration and field-dependent mobility models are considered. Shockley-Read-Hall (SRH) and Auger generation-recombination models are contemplated to acknowledge the lifetime of constant minority carriers and large current density. The simulation temperature was initially $300 \mathrm{~K}$ for the proposed device and then observations were also taken at different temperatures to compare the effect on different device parameters.

The variation of the electron-hole concentration and the energy band diagram of the proposed DL-NT-FET is illustrated in Fig. 2 for OFF-state conditions (VGS=OV and VDS=1V) and ON-state conditions (VGS=1V and VDS=1V) respectively. As it can be noticed from Fig. 2(a) that the concentration of the electrons in the channel region goes high from the low and the holes concentration goes low from high when the device comes into the ON-state condition from OFF-state condition due to the formation of the inversion layer of the electrons and depletion of the holes from the channel [19]. The energy levels of the conduction band and valance band are high in the OFF-state condition as compared to the energy levels of the conduction band and valance band in the ON-state condition Fig. 2(b). The bands bend downwards in ON-state because when a positive gate voltage is applied in the polysilicon gate, the Fermi potential (potential between Fermi level and intrinsic Fermi level) decreases due to an increase in the surface potential, and this result in large electron carriers in the intrinsic (initially) channel region [20]. The introduction of these high electrons shifts the conduction band down towards the Fermi level. 
Simultaneously the holes are also de-voided from the channel due to positive potential on the gate therefore the valance band also shifts downwards away from the Fermi level.

Fig. 3 depicts the variation of electric field and potential in the OFF-state and ON-state conditions. It can be seen from Fig. 3(a) that the electric field uplifts in the interface of source-channel and suppressed at the interface of channel-drain when the device comes in the ON-state condition from the OFF-state, this is due to the application of the positive gate voltage along with the drain voltage [21]. The low electric field at the channel-drain interface indicates less control of the drain to the channel and hence the DL-NT-FET implies low short channel effects. On the other hand, the surface potential goes high in the ON-state as intended due to more bending in the energy bands, at this stage the surface becomes doubled that of Fermi potential.

Fig. 4 shows the variation of the electron mobility and electron-hole pair recombination rate of the DL-NTFET in OFF-state and On-state conditions. The electron mobility goes lower at the source-channel interface and becomes higher at the channel-drain interface Fig. 4(a). It is because the electric field is higher at the source-channel interface and lower at the channel-drain interface as is seen in Fig. 3(a). It can be observed from Fig. 4(b) that the recombination rate of the electron-hole pairs is zero in the channel region ON-state condition of the device channel region.

The drain current variation with channel length is plotted in Fig. 5(a) and its extracted performance parameters are listed in Table 1, it is noted that on increasing the channel length the ION/IOFF ratio enhances while the threshold voltage and Average sub-threshold slope (AVSS) of the DL-NT-FET reduce. An augmentation of 2 orders in the ION/IOFF ratio and a reduction of $22 \%$ and $13 \%$ in the threshold voltage and AVSS respectively have been recorded on increasing the channel length from $15 \mathrm{~nm}$ to 35 $\mathrm{nm}$. The impact of the gate work function variation on the transfer characteristics of the DL-NT-FET is shown in Fig. 5(b) and various device properties that are extracted from its transfer characteristics are mentioned in Table 2. It can be observed that the OFF-current (IOFF) reduces significantly as compare to the ON-current (ION) and therefore the ION/IOFF ratio enhances on increasing the gate work function. The threshold voltage also decreases on augmenting the gate work function whereas the AVSS initially decreases up to a work function of $4.5 \mathrm{eV}$ but then starts increasing. Six orders increment in the ION/IOFF ratio and an $81 \%$ reduction in the threshold voltage has been observed when the gate work function is augmented from $4.3 \mathrm{eV}$ to $4.7 \mathrm{eV}$. Fig. 5(c) exhibits the variation of ID-VGS characteristics with temperature and the various performance parameters at different temperatures are summarized in Table 3 . The IOFF value increases significantly on increasing the temperature while the small degradation in the ION has been noticed as a result of mobility degradation and velocity saturation. And therefore 6 orders diminution in the ION/IOFF ratio is noticed when the temperature is increased from $200 \mathrm{~K}$ to $450 \mathrm{~K}$. threshold voltage and the AVSS both are rose with the temperature. The threshold voltage rises $92 \%$ while the AVSS rises $114 \%$ on increasing the temperature from $200 \mathrm{~K}$ to $450 \mathrm{~K}$. The effect of nanotube radius on the transfer characteristics and hence on various performance parameters are depicted in Fig. 5(d) and Table 4 respectively. On enhancing the radius of the nanotube of the DL-NT-FET, the ION and IOFF both are increasing but the IOFF increases more rapidly as compare to the ION and therefore the ION/IOFF 
ratio reduces. The ION/IOFF ratio is degraded by 3 orders on augmenting the radius from $8 \mathrm{~nm}$ to $16 \mathrm{~nm}$. The threshold voltage and AVSS are increased by increasing the radius of the nanotube; there is an increment of $47 \%$ in threshold voltage whereas an increment of $19 \%$ in the AVSS has been observed while increasing the radius of the nanotube from $8 \mathrm{~nm}$ to $16 \mathrm{~nm}$.

Table 1

Effect of gate work-functions on various device performance parameters of DL-NT-FET

\begin{tabular}{|llllll|}
\hline $\begin{array}{l}\text { Gate-work function } \\
(\mathrm{eV})\end{array}$ & $\begin{array}{l}\text { AVSS } \\
(\mathrm{mV} / \text { decade })\end{array}$ & $\begin{array}{l}\text { Threshold } \\
\text { voltage }(\mathrm{V})\end{array}$ & $\begin{array}{l}\mathrm{I}_{\mathrm{ON}} \\
(\mathrm{A} / \mathrm{m})\end{array}$ & $\begin{array}{l}\mathrm{I}_{\mathrm{OFF}} \\
(\mu \mathrm{A} / \mathrm{m})\end{array}$ & $\mathrm{I}_{\mathrm{ON}} / \mathrm{I}_{\mathrm{OFF}}$ \\
\hline 4.3 & 70.7 & 0.494785 & 41.29 & 930287 & 44.3891 \\
\hline 4.4 & 68.7 & 0.394785 & 38.03 & 52766.2 & 720.727 \\
\hline 4.5 & 68.3 & 0.294785 & 34.53 & 1935.24 & 17845.3 \\
\hline 4.6 & 68.8 & 0.194785 & 30.93 & 64.15 & 482264 \\
\hline 4.7 & 71 & 0.0947849 & 27.21 & 2.04 & 13316400 \\
\hline
\end{tabular}

Table 2

Various device performance parameters at the different channel length of nanotube of DL-NT-FET

\begin{tabular}{|llllll|}
\hline $\begin{array}{l}\text { Channel length } \\
(\mathrm{nm})\end{array}$ & $\begin{array}{l}\text { AVSS } \\
(\mathrm{mV} / \text { decade })\end{array}$ & $\begin{array}{l}\text { Threshold } \\
\text { voltage }(\mathrm{V})\end{array}$ & $\begin{array}{l}\mathrm{I}_{\mathrm{ON}} \\
(\mathrm{A} / \mathrm{m})\end{array}$ & $\begin{array}{l}\mathrm{I}_{\mathrm{OFF}} \\
(\mu \mathrm{A} / \mathrm{m})\end{array}$ & $\mathrm{I}_{\mathrm{ON}} / \mathrm{I}_{\mathrm{OFF}}$ \\
\hline 15 & 74.58 & 0.219144 & 31.15 & 367.3 & 84711.9 \\
\hline 20 & 68.8 & 0.194785 & 30.93 & 64.15 & 482264 \\
\hline 25 & 67.7 & 0.18286 & 30.81 & 21.95 & 1403350 \\
\hline 30 & 66.0 & 0.176297 & 30.70 & 11.26 & 2725660 \\
\hline 35 & 64.57 & 0.170009 & 30.57 & 7.38 & 4141140 \\
\hline
\end{tabular}

Table 3

Variation in the device performance parameters at various temperature conditions 


\begin{tabular}{|llllll|}
\hline $\begin{array}{l}\text { Temperature } \\
(\mathrm{K})\end{array}$ & $\begin{array}{l}\text { AVSS } \\
(\mathrm{mV} / \text { decade })\end{array}$ & $\begin{array}{l}\text { The threshold } \\
\text { voltage }(\mathrm{V})\end{array}$ & $\begin{array}{l}\mathrm{I}_{\mathrm{ON}} \\
(\mathrm{A} / \mathrm{m})\end{array}$ & $\begin{array}{l}\mathrm{I}_{\mathrm{OFF}}(\mathrm{A} / \\
\mu \mathrm{m})\end{array}$ & $\mathrm{I}_{\mathrm{ON}} / \mathrm{I}_{\mathrm{OFF}}$ \\
\hline 200 & 48 & 0.14432 & 31.9 & 0.024 & 1287990000 \\
\hline 250 & 58.6 & 0.168484 & 31.44 & 2.68 & 11714000 \\
\hline 300 & 68.8 & 0.194785 & 30.93 & 64.15 & 482264 \\
\hline 350 & 80.7 & 0.222111 & 30.40 & 637.585 & 47691.7 \\
\hline 400 & 89.2 & 0.249834 & 29.83 & 3624.34 & 8232.48 \\
\hline 450 & 103.3 & 0.277697 & 29.22 & 14074.1 & 2076.18 \\
\hline
\end{tabular}

Table 4

Various device performance parameters at the different radius of nanotube of DL-NT-FET

\begin{tabular}{|llllll|}
\hline Radius $(\mathrm{nm})$ & $\begin{array}{l}\text { AVSS } \\
(\mathrm{mV} / \text { decade })\end{array}$ & $\begin{array}{l}\text { The threshold } \\
\text { voltage }(\mathrm{V})\end{array}$ & $\begin{array}{l}\mathrm{I}_{\mathrm{ON}} \\
(\mathrm{A} / \mathrm{m})\end{array}$ & $\begin{array}{l}\mathrm{I}_{\mathrm{OFF}} \\
(\mu \mathrm{A} / \mathrm{m})\end{array}$ & $\mathrm{I}_{\mathrm{ON}} / \mathrm{I}_{\mathrm{OFF}}$ \\
\hline 8 & 67.5 & 0.178221 & 24.17 & 13.05 & 1852290 \\
\hline 10 & 68.8 & 0.194785 & 30.93 & 64.15 & 482264 \\
\hline 12 & 72.2 & 0.212351 & 37.72 & 298.04 & 126582 \\
\hline 14 & 78.1 & 0.231078 & 44.63 & 1308.17 & 34118 \\
\hline
\end{tabular}

Fig. 6 (a) shows the variation of transconductance with VGS at different channel lengths which is a specific figure of merit of a FET and it measures the precision in the conversance of gate voltage into the current. It is noticed from Fig. 6(a) that the transconductance increases with the increasing channel length (up to $25 \mathrm{~nm}$ ) then it remains constant. The transconductance increases with the channel length because of higher change in drain current is obtained at the large channel length with a small change in gate voltage. At the $25 \mathrm{~nm}$ channel length, the obtained value of the gm is $5.2 \times 10-5 \mathrm{~S} / \mu \mathrm{m}$ at the gate voltage of $0.45 \mathrm{~V}$. Fig. $6(\mathrm{~b})$ depicts the changes in the gate to source capacitance (Cgs) and drain to source capacitance (Cgd) with the gate voltage. A proliferation of $39 \%$ is observed in the Cgs at the VGS of $0.45 \mathrm{~V}$ on increasing the channel length from $20 \mathrm{~nm}$ to $35 \mathrm{~m}$ whereas no significant changes are observed in the Cgd for the same increment in the channel length.

The behavior of drain current concerning VDS for different values of VGS at the channel length of $20 \mathrm{~nm}$ and $35 \mathrm{~nm}$ has been shown in Fig. 7(a), where it is again found that ID is more for channel length $20 \mathrm{~nm}$ than that of for channel length $35 \mathrm{~nm}$. Moreover, it is also noticed that at smaller VGS, the saturation in 
the drain current occurs earlier as compare to the large VGS; this is because of limited space-charge inversion in the channel which implies greater gate control over the channel. Fig. 7(b) shows how the output conductance (gds) varies with VDS where VGS is fixed at $1 \mathrm{~V}$ and readings for $20 \mathrm{~nm}$ and $35 \mathrm{~nm}$ channels are taken. It is well known that gds is a crucial parameter in determining the intrinsic gain of a device and for better gain, it should be low. It can be seen from Fig. 7(b) that its value does not change with the channel length and the obtained value of the gds for the designed DL-NT-FET is $3.4 \times 10-5(\mathrm{~S} / \mu \mathrm{m})$.

The output characteristics (ID-VDS) at the various temperatures are plotted in Fig. 8(a) and it is observed that the drain current decreases with an increase in the temperature. The ID decreases with the temperature because scattering phenomena increase with the temperature and this results in low carrier mobility in the channel and hence low drain current at the higher temperature [22]. The ID degrades $15 \%$ on increasing the temperature from $200 \mathrm{~K}$ to $400 \mathrm{~K}$. Fig. 8(b) shows the variation of the output conductance (gds) with the VDS at different temperature and it is found that gds also degrades on increasing the temperature and it is due to the reduction of ID at the higher temperature.

The impact of the nanotube radius in the output characteristics and output transconductance is plotted in Fig. 9(a) and Fig. 9(b) respectively and it is found that both the parameters are increasing with augmenting the radius. The ID enhances the increasing radius because a large radius renders large space charges in the inversion condition and the large drain current implies the higher output transconductance. An increment of $100 \%$ in the drain current and $137 \%$ in the output transconductance has been noted when the radius of the nanotube increases from $8 \mathrm{~nm}$ to $16 \mathrm{~nm}$.

\section{Conclusion}

This manuscript covers a proposed dopingless nanotube FET structure. Different characteristics of the device have been examined by TCAD simulated results. The obtained AVSS for $20 \mathrm{~nm}$ channel length, the radius of $10 \mathrm{~nm}$ simulated at $300 \mathrm{~K}$ is $68.80 \mathrm{mV} /$ decade which has much scope of improvement by optimization of the device. A comparative analysis of transfer characteristics (ID-VGS), transconductance (gm), gate capacitances (Cgs and Cgd), output characteristics (ID-VDS), and output conductance (gds), has been made by varying the channel length $(\mathrm{Lg})$, radius $(R)$, gate work function $(\Phi)$ and temperature. An increment of $100 \%$ in the drain current and $137 \%$ in the output transconductance has been noted when the radius of the nanotube increases from $8 \mathrm{~nm}$ to $16 \mathrm{~nm}$. The gate capacitances Cgs and Cgd are higher for the longer channel. On increasing the temperature from $300 \mathrm{k}$ to $400 \mathrm{~K}$, the output conductance is found to decrease as well as the drain current gets saturated to a lower value whereas, by decreasing temperature to $200 \mathrm{~K}$, the opposite happens. An augmentation of six orders in the ION/IOFF ratio and $81 \%$ reduction in the threshold voltage has been observed when the gate work function is enhanced from $4.3 \mathrm{eV}$ to $4.7 \mathrm{eV}$. The ION/IOFF decreases with a temperature rise, there are 6 orders diminution in the ION/IOFF ratio is noticed when the temperature is increased from $200 \mathrm{~K}$ to $450 \mathrm{~K}$. The threshold voltage and the AVSS both are rose with the temperature. The threshold voltage rises $92 \%$ while the AVSS rises 
$114 \%$ on increasing the temperature from $200 \mathrm{~K}$ to $450 \mathrm{~K}$. The transconductance curve has the highest peak value for the longest channel. It can thus be concluded that, after a thorough investigation of the behavior of the proposed dopingless nanotube FET parameters under several variations, it can be well understood that there are a good number of challenges to get the optimized results.

\section{Declarations}

\section{Funding statement:}

No funding was received to assist with the preparation of this manuscript.

\section{Conflict of Interest:}

The authors declare that they have no conflict of interest.

\section{Author contributions:}

All authors have equally participated in the preparing of the manuscript during implementation of ideas, findings results, and writing of the manuscript.

\section{Availability of data and material:}

current submission does not contain the pool data of the manuscript but the data used in the manuscript will be provided on request.

\section{Compliance with ethical standards:}

The Authors accepted principles of ethical standard and they have no conflict of interest.

\section{Consent to participate:}

Informed consent.

\section{Consent for Publication:}

Consent is granted.

\section{Acknowledgments:}


Not Applicable.

\section{References}

1. Huang, J.S.T., and Schrankler, J.W., 1987. Switching characteristics of scaled CMOS circuits at 77 K. IEEE transactions on electron devices, 34(1), pp.101-106.

2. Kong, L., Chen, Y. and Liu, Y., 2020. Recent progresses of NMOS and CMOS logic functions based on two-dimensional semiconductors. Nano Research, pp.1-16.

3. Singh, J. and Raj, B., 2019. Design and Investigation of 7T2M-NVSRAM With Enhanced Stability and Temperature Impact on Store/Restore Energy. IEEE Transactions on Very Large Scale Integration (VLSI) Systems, 27(6), pp.1322-1328.

4. Pourghaderi, M.A., Pham, A.T., llatikhameneh, H., Kim, J., Park, H.H., Jin, S., Chung, W.Y., Choi, W., Maeda, S. and Lee, K.H., 2017. Universality of Short-Channel Effects on Ultrascaled MOSFET Performance. IEEE Electron Device Letters, 39(2), pp.168-171.

5. Ribeiro, T.A. and Pavanello, M.A., 2020, February. Analysis of the Electrical Parameters in SOI n-type Junctionless Nanowire Transistors at High Temperatures. In 2020 IEEE Latin America Electron Devices Conference (LAEDC) (pp. 1-4). IEEE.

6. Sahu, C. and Parmar, J.S., 2017, July. Analog/rf performance comparison of junctionless and dopingless field effect transistor. In 2017 International Conference on Computer, Communications and Electronics (Comptelix) (pp. 606-611). IEEE.

7. Chebaki, E., Djeffal, F., Ferhati, H. and Bentrcia, T., 2016. Improved analog/RF performance of double gate junctionless MOSFET using both gate material engineering and drain/source extensions. Superlattices and Microstructures, 92, pp.80-91.

8. Kuhn, K., 2018. Cmos and beyond cmos: Scaling challenges. In High Mobility Materials for CMOS Applications (pp. 1-44). Woodhead Publishing.

9. Trojman, L., Ragnarsson, L.Å. and Collaert, N., 2019. Mobility extraction for short channel UTBBFDSOI MOSFETs under back bias using an accurate inversion charge density model. Solid-State Electronics, 154, pp.24-30.

10. Shan, C., Wang, Y. and Bao, M.T., 2016. A charge-plasma-based transistor with induced graded channel for enhanced analog performance. IEEE Transactions on electron devices, 63(6), pp.22752281.

11. Kumar, N., Amin, S.I. and Anand, S., 2020. Design and Performance Optimization of Novel Core-Shell Dopingless GAA-Nanotube TFET With Si 0.5 Ge 0.5-Based Source. IEEE Transactions on Electron Devices, 67(3), pp.789-795.

12. Gupta, A.K. and Raman, A., 2020. Performance analysis of electrostatic plasma-based dopingless nanotube TFET. Applied Physics A, 126(7), pp.1-10.

13. Mushtaq, U., Kumar, N., Anand, S. and Amin, I., 2020. Design and Performance Analysis of Core-Shell Dual Metal-Dual Gate Cylindrical GAA Silicon Nanotube-TFET. Silicon, 12(10), pp.2355-2363. 
14. Tayal, S. and Nandi, A., 2018. Optimization of gate-stack in junctionless Si-nanotube FET for analog/RF applications. Materials Science in Semiconductor Processing, 80, pp.63-67.

15. Liu, M., Lentz, F., Trellenkamp, S., Hartmann, J.M., Knoch, J., Grützmacher, D., Buca, D. and Zhao, Q.T., 2020. Diameter Scaling of Vertical Ge Gate-All-Around Nanowire pMOSFETs. IEEE Transactions on Electron Devices, 67(7), pp.2988-2994.

16. Raad, B.R., Sharma, D., Kondekar, P., Nigam, K. and Yadav, D.S., 2016. Drain work function engineered doping-less charge plasma TFET for ambipolar suppression and RF performance improvement: a proposal, design, and investigation. IEEE Transactions on Electron Devices, 63(10), pp.3950-3957.

17. Oks, E.M., 1992. Physics and technique of plasma electron sources. Plasma Sources Science and Technology, 1(4), p.249.

18. Gusev, E.P., Narayanan, V. and Frank, M.M., 2006. Advanced high-k dielectric stacks with polySi and metal gates: Recent progress and current challenges. IBM Journal of Research and Development, 50(4.5), pp.387-410.

19. Sallese, J.M., Bucher, M., Krummenacher, F. and Fazan, P., 2003. Inversion charge linearization in MOSFET modeling and rigorous derivation of the EKV compact model. Solid-State Electronics, 47(4), pp.677-683.

20. Kang, S.M. and Leblebici, Y., 2003. CMOS digital integrated circuits. Tata McGraw-Hill Education.

21. Assaderaghi, F., Parke, S., Sinitsky, D., Bokor, J., Ko, P.K. and Hu, C., 1994. A dynamic threshold voltage MOSFET (DTMOS) for very low voltage operation. IEEE Electron Device Letters, 15(12), pp.510-512.

22. Blatt, F.J., 1957. Scattering of carriers by ionized impurities in semiconductors. Journal of Physics and Chemistry of Solids, 1(4), pp.262-269.

\section{Figures}

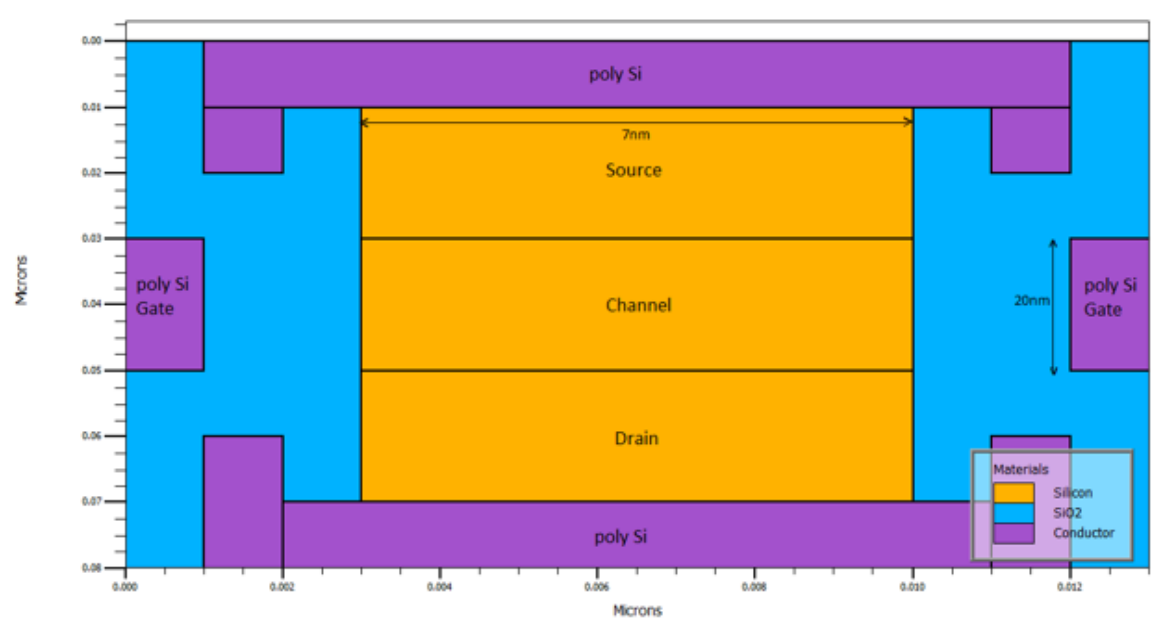

(a)

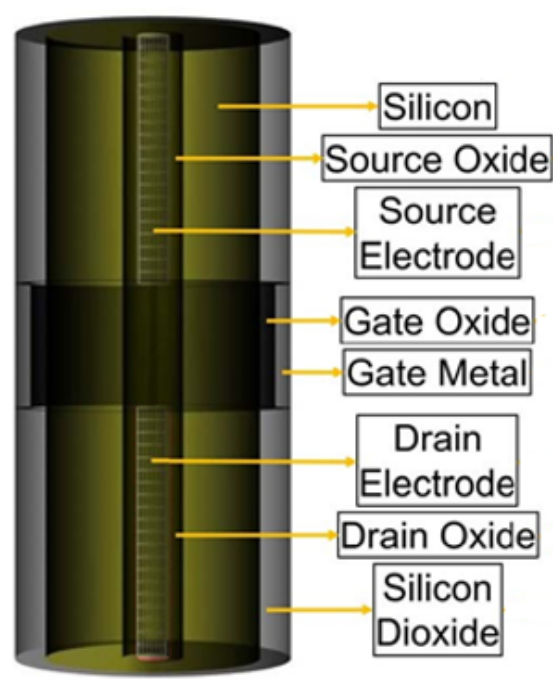

(b) 
Figure 1

(a) 2-D cross-sectional view of the proposed device (b) 3-D view of the proposed device
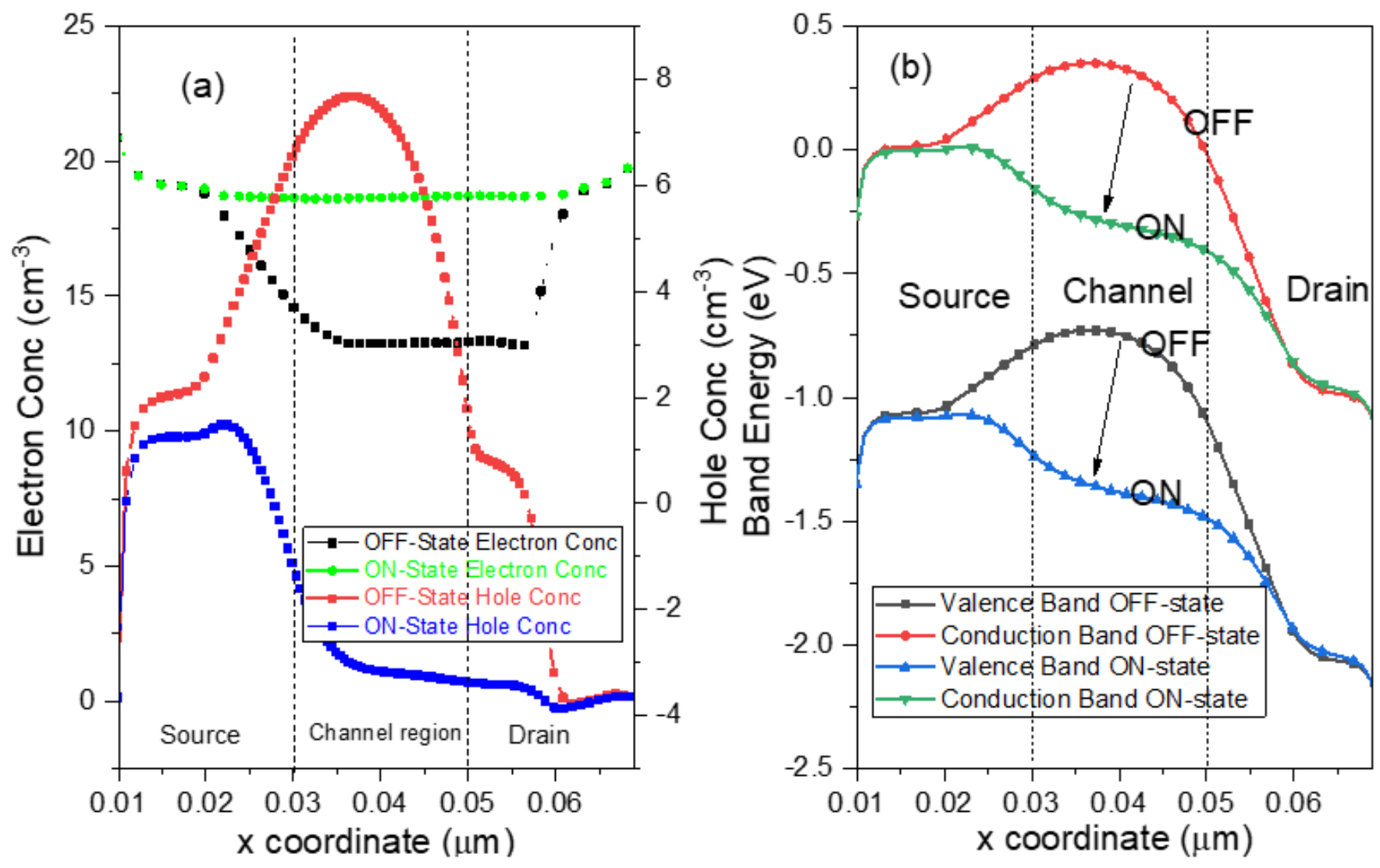

Figure 2

Variation of (a) Electrons and holes carrier concentration and (b) the energy band diagram of DL-NT-FET in OFF condition (VGS $=0 \mathrm{~V}$ and VDS $=1 \mathrm{~V}$ ) and in $\mathrm{ON}$ condition ( $\mathrm{VGS}=1 \mathrm{~V}$ and $\mathrm{VDS}=1 \mathrm{~V}), \mathrm{x}$ coordinate shows the distance from the source to drain along the channel 

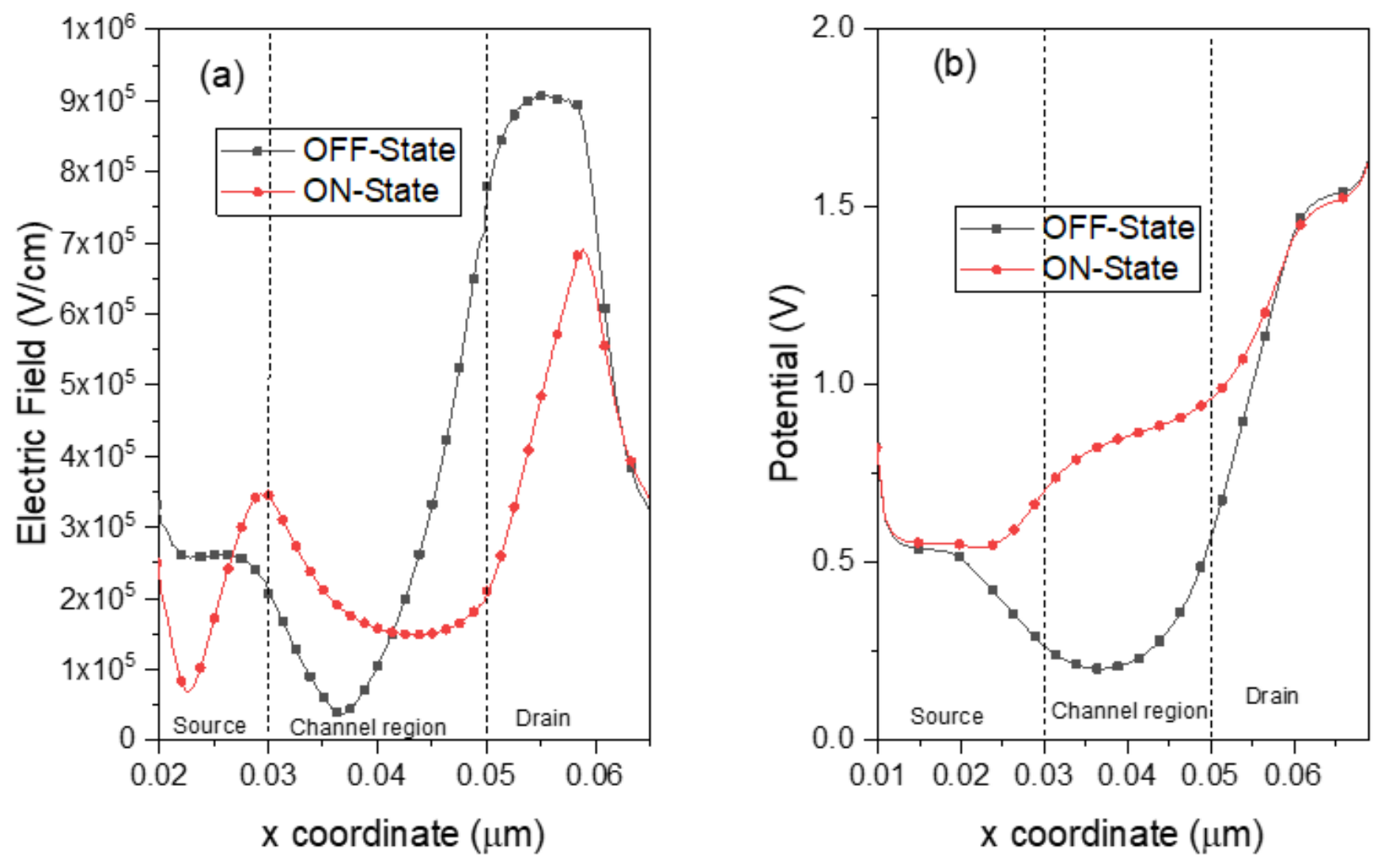

\section{Figure 3}

Variation of (a) Electric Field and (b) Potential of DL-NT-FET in OFF condition (VGS=0V and VDS $=1 \mathrm{~V}$ ) and in $\mathrm{ON}$ condition (VGS=1V and VDS=1V), $\mathrm{x}$ coordinate shows the distance from the source to drain along the channel 

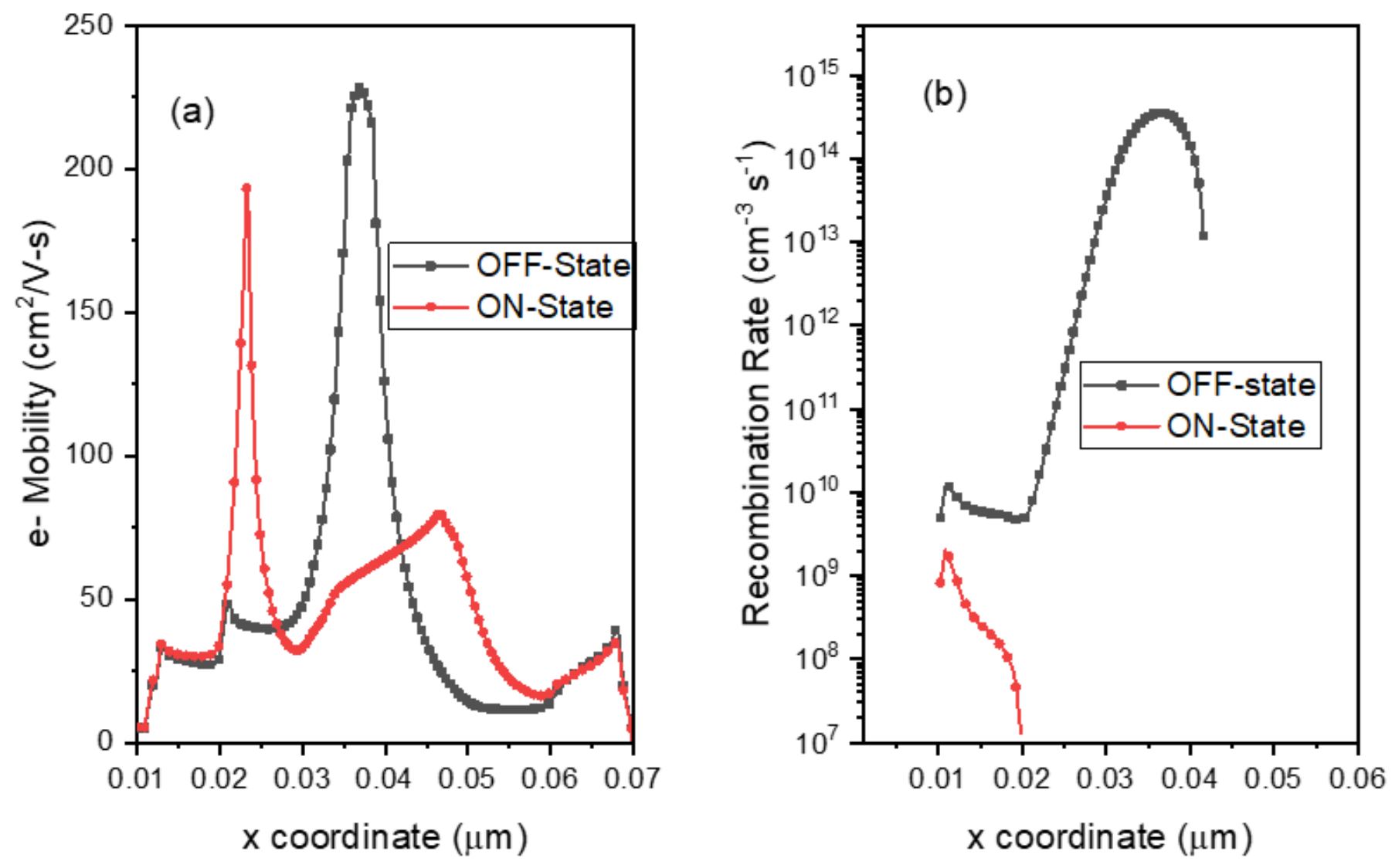

Figure 4

Variation of (a) Electron Mobility and (b) Electro-hole recombination rate of DL-NT-FET in OFF condition (VGS=0V and VDS=1V) and in ON condition (VGS=1V and VDS=1V), $x$ coordinate shows the distance from the source to drain along the channel 

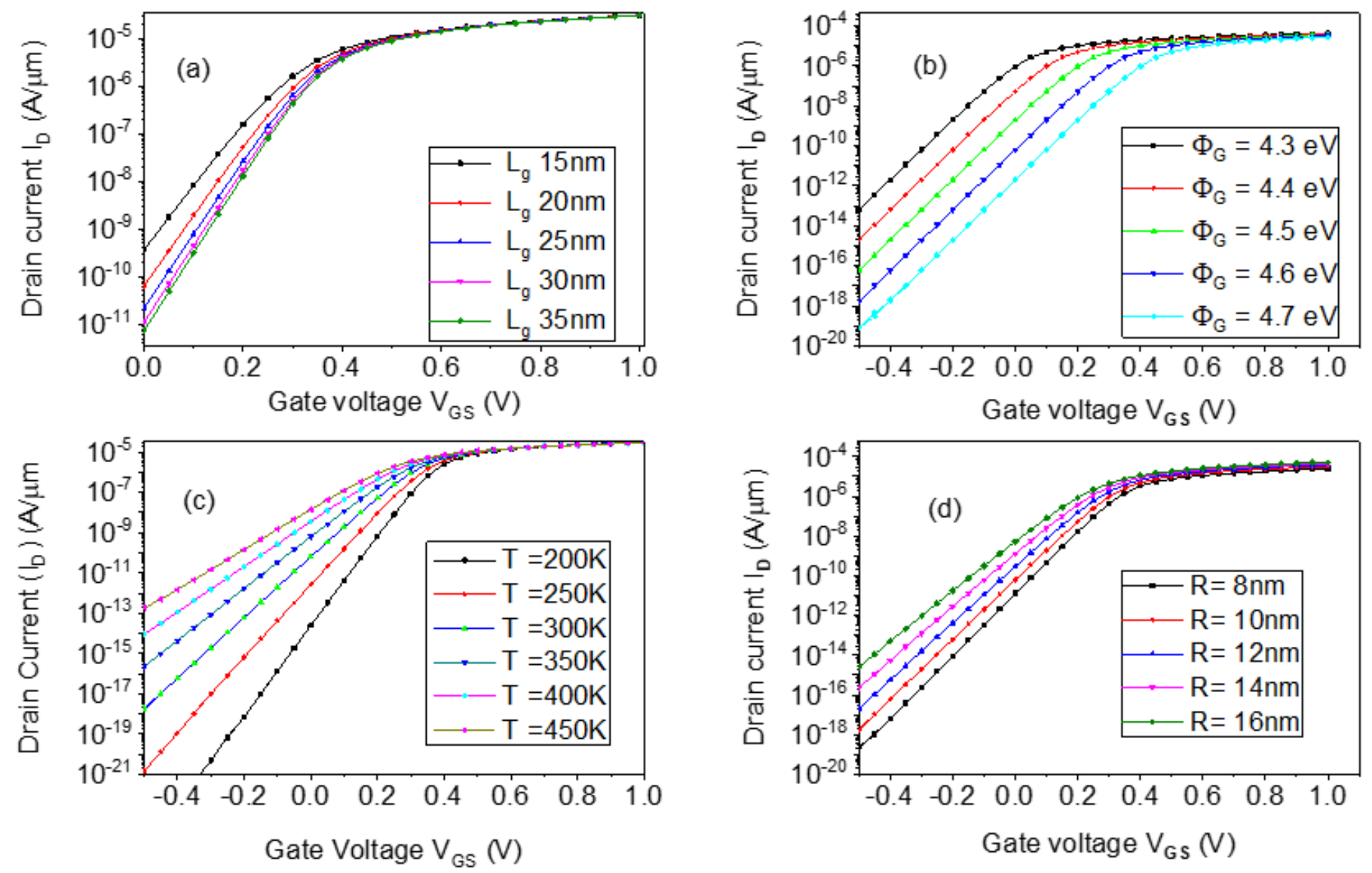

Figure 5

Variation of (ID-VGS) characteristics of the DL-NT-FET at VDS=1V for (a) the varying the channel length $(\mathrm{Lg})(\mathrm{b})$ the varying the gate electrode work function ( $(\mathrm{G})$ (c) at various temperature $(\mathrm{T})$, and (d) the varying the radius $(\mathrm{R})$ of the DL-NT-FET. 

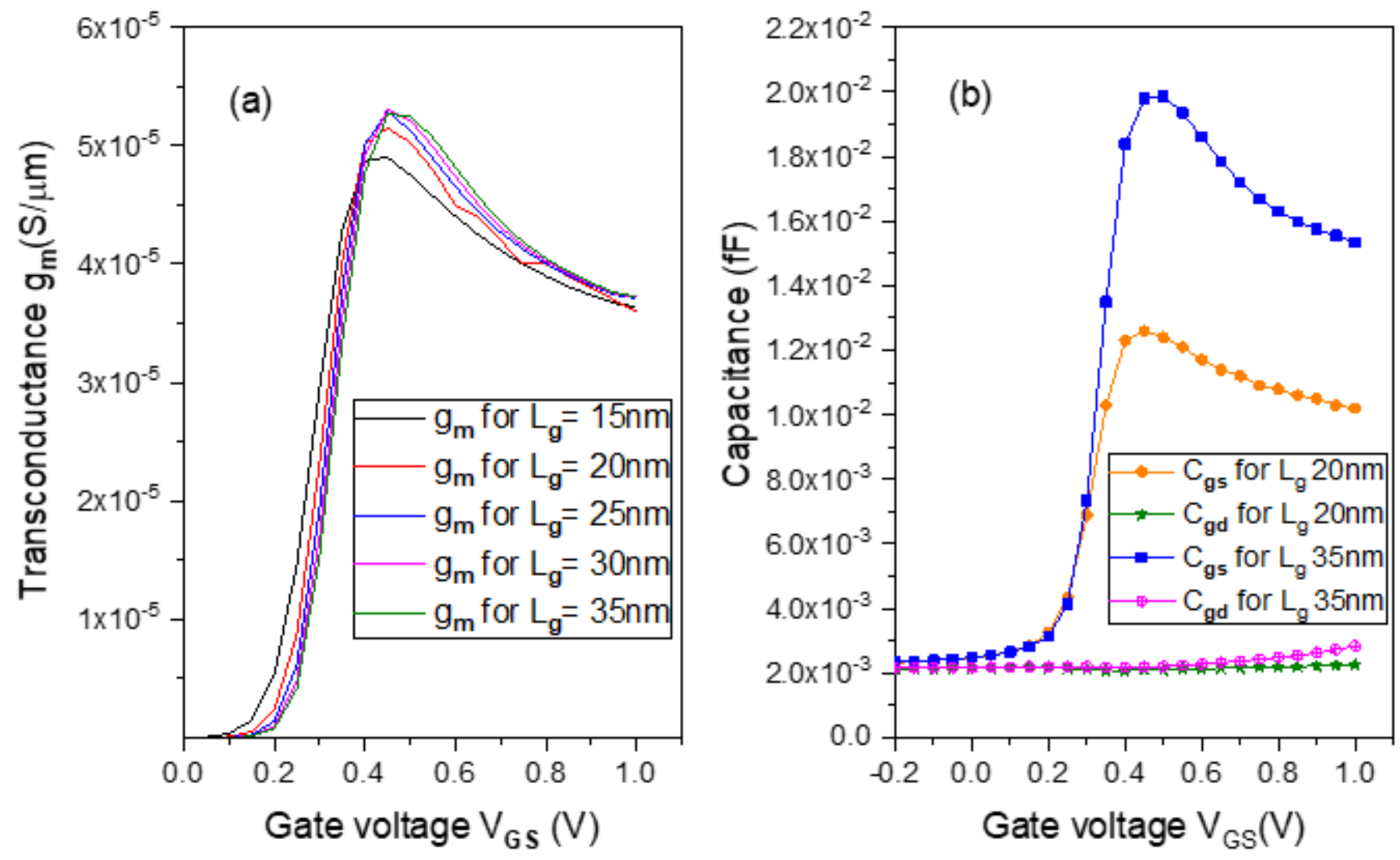

Figure 6

Variation of (a) transconductance ( $\mathrm{gm}$ ) and (b) gate to source capacitance (Cgs) and gate to drain capacitance (Cgd) with gate voltage at various channel length and VDS=1V
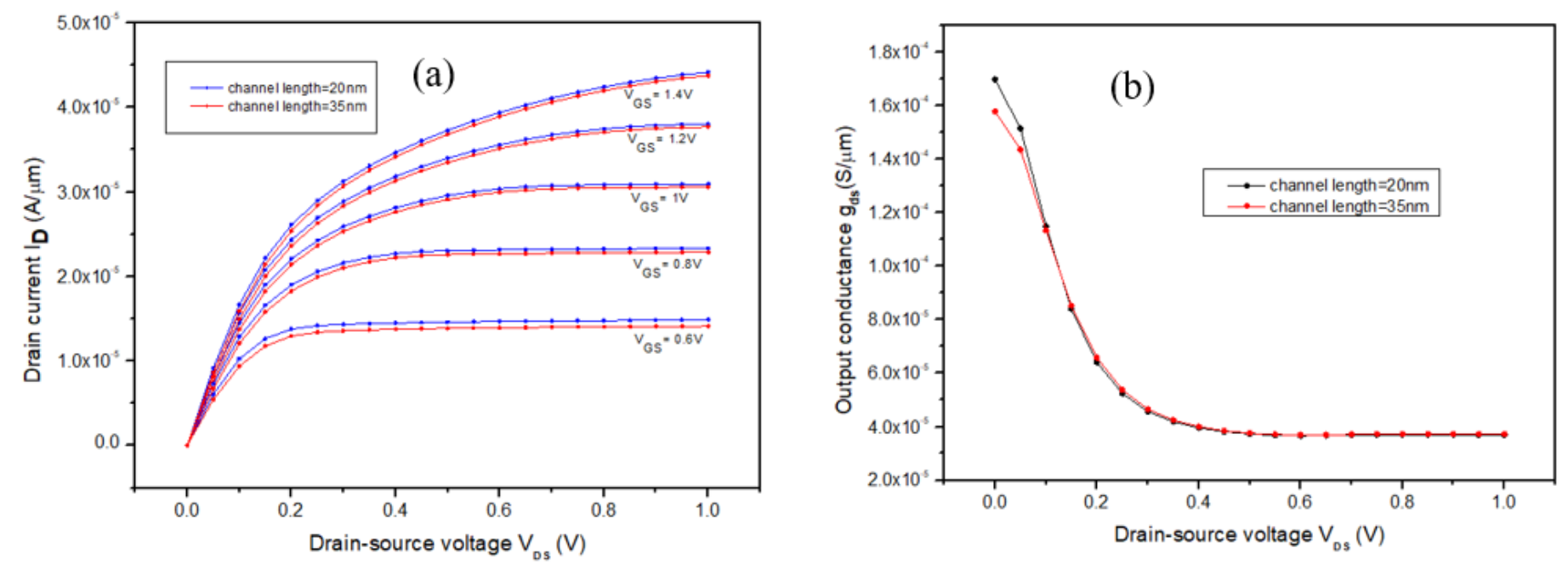

Figure 7 
(a) Output characteristics for channel lengths $20 \mathrm{~nm}$ and $35 \mathrm{~nm}$ at different VGS. (b) Output conductance vs VDS for channel lengths $20 \mathrm{~nm}$ and $35 \mathrm{~nm}$ at VGS $=1 \mathrm{~V}$.
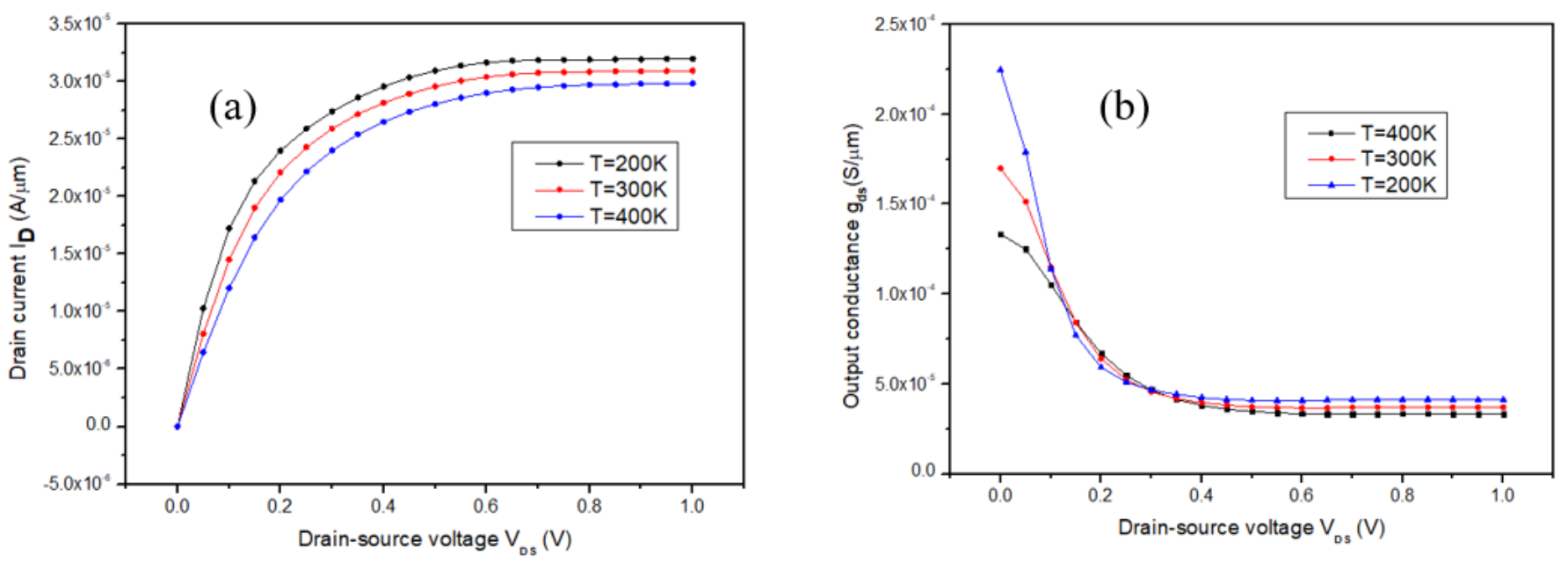

\section{Figure 8}

Variation of (a) output characteristics (IDS-VDS) at different temperatures and (b) output conductance for different temperatures (both at VGS=1V)
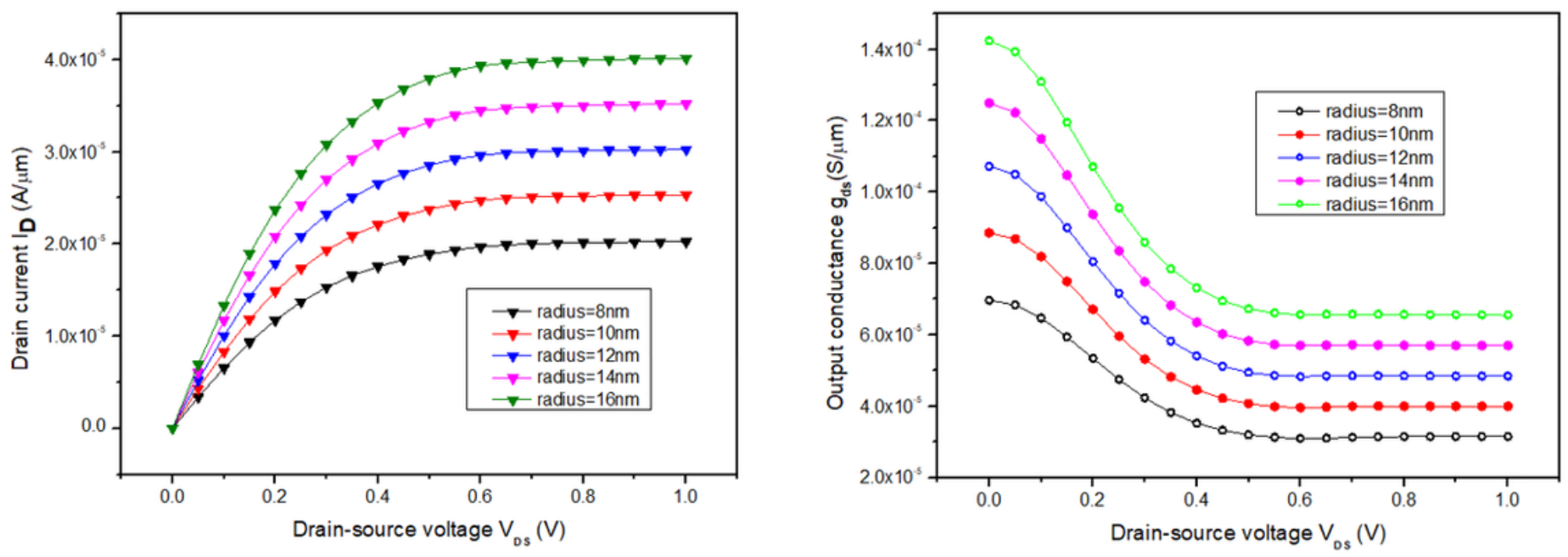

Figure 9

(a) Output characteristics for different radius (b) Output conductance Vs. VDS for the different radius (both at VGS=1V) 